\title{
Double almost lacunary statistical convergence of order $\alpha$
}

\section{Ekrem Savaş*}

\section{"Correspondence:}

ekremsavas@yahoo.com

Department of Mathematics,

Istanbul Commerce University,

Üsküdar, Istanbul, Turkey

\begin{abstract}
In this paper, we define and study lacunary double almost statistical convergence of order $\alpha$. Further, some inclusion relations have been examined. We also introduce a new sequence space by combining lacunary double almost statistical convergence and Orlicz function.
\end{abstract}

MSC: Primary 40B05; secondary 40C05

Keywords: statistical convergence; Orlicz function; double statistical convergence of order $\alpha$; lacunary statistical convergence; double almost statistical convergence

\section{Introduction}

The notion of convergence of a real sequence was extended to a statistical convergence by Fast [1] (see also Schoenberg [2]) as follows. If $\mathbb{N}$ denotes the set of natural numbers and $K \subset \mathbb{N}$, then $K(m, n)$ denotes the cardinality of the set $K \cap[m, n]$. The upper and lower natural density of the subset $K$ is defined by

$$
\bar{d}(K)=\lim _{n \rightarrow \infty} \sup \frac{K(1, n)}{n} \text { and } \quad \underline{d}(K)=\lim _{n \rightarrow \infty} \inf \frac{K(1, n)}{n} .
$$

If $\bar{d}(K)=\underline{d}(K)$, then we say that the natural density of $K$ exists, and it is denoted simply by $d(K)$. Clearly $d(K)=\lim _{n \rightarrow \infty} \frac{K(1, n)}{n}$.

A sequence $x=\left(x_{k}\right)$ of real numbers is said to be statistically convergent to $L$ if for arbitrary $\epsilon>0$, the set $K(\epsilon)=\left\{k \in \mathbb{N}:\left|x_{k}-L\right| \geq \epsilon\right\}$ has a natural density zero.

Statistical convergence turned out to be one of the most active areas of research in summability theory after the works of Fridy [3] and Šalát [4]. For some very interesting investigations concerning statistical convergence, one may consult the papers of Cakalli [5], Miller [6], Maddox [7] and many others, where more references on this important summability method can be found.

On the other hand, in $[8,9]$, a different direction was given to the study of statistical convergence, where the notion of statistical convergence of order $\alpha, 0<\alpha<1$ was introduced by replacing $n$ by $n^{\alpha}$ in the denominator in the definition of statistical convergence. It was observed in [8] that the behaviour of this new convergence was not exactly parallel to that of statistical convergence, and some basic properties were obtained. One can also see [10] for related works.

In this paper, we define and study lacunary double almost statistical convergence of or$\operatorname{der} \alpha$. Also some inclusion relations have been examined.

2013 Savas: licensee Springer. This is an Open Access article distributed under the terms of the Creative Commons Attribution License (http://creativecommons.org/licenses/by/2.0), which permits unrestricted use, distribution, and reproduction in any medium, provided the original work is properly cited. 
Let $w_{2}$ be the set of all real or complex double sequences. By the convergence of a double sequence, we mean the convergence on the Pringsheim sense, that is, double sequence $x=\left(x_{i j}\right)$ has a Pringsheim $\operatorname{limit} L$, denoted by $P-\lim x=L$, provided that given $\epsilon>0$, and there exists $N \in \mathbb{N}$ such that $\left|x_{i j}-L\right|<\epsilon$ whenever $i, j \geq N$. We shall describe such an $x$ more briefly as ' $P$-convergent' (see, [11]). We denote by $c_{2}$ the space of $P$-convergent sequences. A double sequence $x=\left(x_{i j}\right)$ is bounded if $\|x\|=\sup _{i, j \geq 0}\left|x_{i j}\right|<\infty$. Let $l_{2}^{\infty}$ and $c_{2}^{\infty}$ be the set of all real or complex bounded double sequences and the set bounded and convergent double sequences, respectively. Moricz and Rhoades [12] defined the almost convergence of double sequence as follows: $x=\left(x_{i j}\right)$ is said to be almost convergent to a number $L$ if

$$
P-\lim _{p, q \rightarrow \infty} \sup _{m, n}\left|\frac{1}{(p+1)(q+1)} \sum_{i=m}^{m+p} \sum_{j=n}^{n+q} x_{i j}-L\right|=0,
$$

that is, the average value of $\left(x_{i j}\right)$ taken over any rectangle

$$
D=\{(i, j): m \leq i \leq m+p, n \leq j \leq n+q\},
$$

tends to $L$ as both $p$ and $q$ tend to $\infty$, and this convergence is uniform in $m$ and $n$. We denote the space of almost convergent double sequence by $\hat{c}_{2}$, as

$$
\hat{c}_{2}=\left\{x=\left(x_{i j}\right): \lim _{k, l \rightarrow \infty}\left|t_{k l p q}(x)-L\right|=0 \text {, uniformly in } p, q\right\}
$$

where

$$
t_{k l p q}(x)=\frac{1}{(k+1)(l+1)} \sum_{i=p}^{k+p} \sum_{j=q}^{l+q} x_{i j}
$$

The notion of almost convergence for single sequences was introduced by Lorentz [13] and some others.

A double sequence $x$ is called strongly double almost convergent to a number $L$ if

$$
P-\lim _{k, l \rightarrow \infty} \frac{1}{(k+1)(l+1)} \sum_{i=p}^{k+p} \sum_{j=q}^{l+q}\left|x_{i j}-L\right|=0, \quad \text { uniformly in } p, q .
$$

By $\left[\hat{c}_{2}\right]$, we denote the space of strongly almost convergent double sequences.

The notion of strong almost convergence for single sequences has been introduced by Maddox [7].

The idea of statistical convergence was extended to double sequences by Mursaleen and Edely [14]. More recent developments on double sequences can be found in [8, 15-18]. For the single sequences; statistical convergence of order $\alpha$ and strongly $p$-Cesàro summability of order $\alpha$ introduced by Çolak [9]. Quite recently, in [10], Çolak and Bektaş generalized this notion by using de la Valée-Poussin mean.

Let $K \subseteq \mathbb{N} \times \mathbb{N}$ be a two-dimensional set of positive integers, and let $K_{m, n}$ be the numbers of $(i, j)$ in $K$ such that $i \leq n$ and $j \leq m$. 
Then the lower asymptotic density of $K$ is defined as

$$
P-\liminf _{m, n} \frac{K_{m, n}}{m n}=\delta_{2}(K)
$$

In the case when the sequence $\left(\frac{K_{m, n}}{m n}\right)_{m, n=1,1}^{\infty, \infty}$ has a limit, we say that $K$ has a natural density and is defined as

$$
P-\lim _{m, n} \frac{K_{m, n}}{m n}=\delta_{2}(K)
$$

For example, let $K=\left\{\left(i^{2}, j^{2}\right): i, j \in \mathbb{N}\right\}$, where $\mathbb{N}$ is the set of natural numbers. Then

$$
\delta_{2}(K)=P-\lim _{m, n} \frac{K_{m, n}}{m n} \leq P-\lim _{m, n} \frac{\sqrt{m} \sqrt{n}}{m n}=0
$$

(i.e., the set $K$ has a double natural density zero).

Mursaleen and Edely [14] presented the notion of a statistical convergence for the double sequence $x=\left(x_{i j}\right)$ as follows: A real double sequence $x=\left(x_{i j}\right)$ is said to be statistically convergent to $L$, provided that for each $\epsilon>0$

$$
P-\lim _{m, n} \frac{1}{m n} \mid\left\{(i, j): i \leq m \text { and } j \leq n,\left|x_{i j}-L\right| \geq \epsilon\right\} \mid=0 \text {. }
$$

We now write the following definition.

The double statistical convergence of order $\alpha$ is defined as follows. Let $0<\alpha \leq 1$ be given. The sequence $\left(x_{i j}\right)$ is said to be statistically convergent of order $\alpha$ if there is a real number $L$ such that

$$
P-\lim _{m n \rightarrow \infty} \frac{1}{(m n)^{\alpha}} \mid\left\{i \leq m \text { and } j \leq n:\left|x_{i j}-L\right| \geq \epsilon\right\} \mid=0
$$

for every $\epsilon>0$, in this, case we say that $x$ is double statistically convergent of order $\alpha$ to $L$. In this case, we write $S_{2}^{\alpha}$ - $\lim x_{i j}=L$. The set of all double statistically convergent sequences of order $\alpha$ will be denoted by $S_{2}^{\alpha}$. If we take $\alpha=1$ in this definition, we can have the previous definition.

By a lacunary $\theta=\left(k_{r}\right) ; r=0,1,2, \ldots$, where $k_{0}=0$, we shall mean an increasing sequence of nonnegative integers with $k_{r}-k_{r-1} \rightarrow \infty$ as $r \rightarrow \infty$. The intervals determined by $\theta$ will be denoted by $I_{r}=\left(k_{r-1}, k_{r}\right]$ and $h_{r}=k_{r}-k_{r-1}$. The ratio $\frac{k_{r}}{k_{r-1}}$ will be denoted by $q_{r}$.

Fridy and Orhan [19] introduced the idea of lacunary statistical convergence for single sequence as follows.

The number sequence $x=\left(x_{i}\right)$ is said to be lacunary statistically convergent to the number $\ell$ if for each $\epsilon>0$,

$$
\lim _{n} \frac{1}{h_{r}}\left|\left\{k \in I_{r}:\left|x_{i}-L\right| \geq \epsilon\right\}\right|=0 .
$$

In this case, we write $S_{\theta}-\lim _{i} x_{i}=\ell$, and we denote the set of all lacunary statistically convergent sequences by $S_{\theta}$. 
Definition 1.1 By a double lacunary $\theta_{r s}=\left\{\left(k_{r} l_{s}\right)\right\}, r, s=0,1,2, \ldots$, where $k_{0}=0$ and $l_{0}=0$, we shall mean two increasing sequences of nonnegative integers with

$$
h_{r}=k_{r}-k_{k-1} \rightarrow \infty \quad \text { as } r \rightarrow \infty
$$

and

$$
\bar{h}_{s}=l_{s}-l_{s-1} \rightarrow \infty \quad \text { as } s \rightarrow \infty .
$$

Let us denote $k_{r s}=k_{r} l_{s}, h_{r s}=h_{r} \bar{h}_{s}$ and the intervals determined by $\theta_{r s}$ will be denoted by $I_{r s}=\left\{(k, l): k_{r-1}<k \leq k_{r}\right.$ and $\left.l_{s-1}<l \leq l_{s}\right\}, q_{r}=\frac{k_{r}}{k_{r-1}}, \bar{q}_{s}=\frac{l_{s}}{l_{s-1}}$, and $q_{r s}=q_{r} \bar{q}_{s}$. We will denote the set of all double lacunary sequences by $\mathbf{N}_{\theta_{r s}}$.

Let $K \subseteq N \times N$ have double lacunary density $\delta_{2}^{\theta}(K)$ if

$$
P-\lim _{r s} \frac{1}{h_{r s}}\left|\left\{(k, l) \in I_{r s}:(k, l) \in K\right\}\right|
$$

exists.

Example 1 Let $\theta=\left\{\left(2^{r}-1,3^{s}-1\right)\right\}$ and $K=\{(k, 2 l): k, l \in N \times N\}$. Then $\delta_{2}^{\theta}(K)=0$. But it is obvious that $\delta_{2}(K)=1 / 2$.

In 2005, Patterson and Savaş [17] studied double lacunary statistical convergence by giving the definition for complex sequences as follows.

Definition 1.2 Let $\theta_{r s}$ be a double lacunary sequence; the double number sequence $x$ is $S_{\theta}^{2}$-convergent to $L$, provided that for every $\epsilon>0$,

$$
P-\lim _{r s} \frac{1}{h_{r s}}\left|\left\{(k, l) \in I_{r s}:\left|x_{k l}-L\right| \geq \epsilon\right\}\right|=0 .
$$

In this case, write $S_{\theta}^{2}-\lim x=L$ or $x_{k l} \stackrel{P}{\rightarrow} L\left(S_{\theta}^{2}\right)$.

More investigation in this direction and more applications of double lacunary and double sequences can be found in [20-22] and [23].

\section{Main results}

In this section, we define lacunary double almost statistically convergent sequences of order $\alpha$. Also we shall prove some inclusion theorems.

We now have the following.

Definition 2.1 Let $0<\alpha \leq 1$ be given. The sequence $x=\left(x_{i j}\right) \in w_{2}$ is said to be $\hat{S}_{\theta_{r s}}^{\alpha}$ statistical convergence of order $\alpha$ if there is a real number $L$ such that

$$
P-\lim _{r s} \frac{1}{h_{r s}^{\alpha}}\left|\left\{(k, l) \in I_{r s}:\left|t_{k l p q}(x)-L\right| \geq \epsilon\right\}\right|=0, \quad \text { uniformly in } p, q \text {, }
$$

where $h_{r s}^{\alpha}$ denote the $\alpha$ th power $\left(h_{r s}\right)^{\alpha}$ of $h_{r s}$. In case $x=\left(x_{i j}\right)$ is $\hat{S}_{\theta_{r s}}^{\alpha}$-statistically convergent of order $\alpha$ to $L$, we write $\hat{S}_{\theta_{r s}}^{\alpha}-\lim x_{i j}=L$. We denote the set of all $\hat{S}_{\theta_{r s}}^{\alpha}$-statistically convergent sequences of order $\alpha$ by $\hat{S}_{\theta_{r s}}^{\alpha}$. 
We know that the $\hat{S}_{\theta_{r s}}^{\alpha}$-statistical convergence of order $\alpha$ is well defined for $0<\alpha \leq 1$, but it is not well defined for $\alpha>1$ in general. It is easy to see by taking $x=\left(x_{i j}\right)$ as fixed.

Definition 2.2 Let $0<\alpha \leq 1$ be any real number, and let $t$ be a positive real number. A sequence $x$ is said to be strongly $\hat{w}_{\theta_{r S}}^{\alpha}(t)$-summable of order $\alpha$, if there is a real number $L$ such that

$$
P-\lim _{r s} \frac{1}{h_{r s}^{\alpha}} \sum_{(k, l) \in I_{r s}}\left|t_{k l p q}(x)-L\right|^{t}=0, \quad \text { uniformly in } p, q .
$$

If we take $\alpha=1$, the strong $\hat{w}_{\theta_{r s}}^{\alpha}(t)$-summability of order $\alpha$ reduces to the strong $\hat{w}_{\theta_{r s}}(t)$ summability.

We denote the set of all strongly $\hat{w}_{\theta_{r s}}^{\alpha}(t)$-summable sequence of order $\alpha$ by $\hat{w}_{\theta_{r s}}^{\alpha}(t)$.

We now state the following theorem.

Theorem 2.1 If $0<\alpha \leq \beta \leq 1$, then $\hat{S}_{\theta_{r s}}^{\alpha} \subset \hat{S}_{\theta_{r s}}^{\beta}$.

Proof Let $0<\alpha \leq \beta \leq 1$. Then

$$
\frac{1}{h_{r s}^{\beta}}\left|\left\{(k, l) \in I_{r s}:\left|t_{k l p q}(x)-L\right| \geq \epsilon\right\}\right| \leq \frac{1}{h_{r s}^{\alpha}}\left|\left\{(k, l) \in I_{r s}:\left|t_{k l p q}(x)-L\right| \geq \epsilon\right\}\right|
$$

for every $\epsilon>0$, and finally, we have that $\hat{S}_{\theta_{r s}}^{\alpha} \subset \hat{S}_{\theta_{r s}}^{\beta}$. This proves the result.

Theorem 2.2 For any lacunary sequences $\theta, \hat{S}_{2}^{\alpha} \subseteq \hat{S}_{\theta_{r s}}^{\alpha}$, if $\liminf q_{r}>1$ and $\liminf \bar{q}_{s}>1$.

Proof Suppose that $\liminf q_{r}^{\alpha}>1$ and $\liminf q_{s}^{\alpha}>1, \liminf q_{r}^{\alpha}=\alpha_{1}$ and $\liminf q_{s}^{\alpha}=\alpha_{2}$, say. Write $\beta_{1}=\left(\alpha_{1}-1\right) / 2$ and $\beta_{2}=\left(\alpha_{2}-1\right) / 2$. Then there exist a positive integer $r_{0}$ and $s_{0}$ such that $q_{r}^{\alpha} \geq 1+\beta_{1}$ for $r \geq r_{0}$ and $q_{s} \geq 1+\beta_{2}$ for $s \geq s_{0}$. Hence for $r \geq r_{0}$, and $s \geq s_{0}$,

$$
\begin{aligned}
h_{r s}^{\alpha} \frac{1}{\left(k_{r} l_{s}\right)^{\alpha}} & =1-\left(\frac{k_{r-1}^{\alpha}}{k_{r}^{\alpha}}\right) \times 1-\left(\frac{l_{s-1}^{\alpha}}{l_{s}^{\alpha}}\right) \\
& =\left(1-\frac{1}{q_{r}^{\alpha}}\right) \times\left(1-\frac{1}{q_{s}^{\alpha}}\right) \\
& \geq 1-\frac{1}{\left(1+\beta_{1}\right)} \times 1-\frac{1}{\left(1+\beta_{2}\right)} \\
& =\frac{\beta_{1}}{1+\beta_{1}} \times \frac{\beta_{2}}{1+\beta_{2}} .
\end{aligned}
$$

Take any $\left(x_{k l}\right) \in \hat{S}_{2}^{\alpha}$, and $\hat{S}_{2}^{\alpha}-\lim _{(k, l) \rightarrow \infty} x_{k l}=L$, say. We prove that $\hat{S}_{\theta_{r s}}^{\alpha}-\lim _{(k, l) \rightarrow \infty} x_{k l}=L$. Then for $r \geq r_{0}$ and $s \geq s_{0}$, we have

$$
\begin{aligned}
& \frac{1}{\left(k_{r} l_{s}\right)^{\alpha}}\left|\left\{k \leq k_{r}, l \leq l_{s}:\left|t_{k l p q}(x)-L\right| \geq \epsilon\right\}\right| \\
& \quad \geq \frac{1}{\left(k_{r} l_{s}\right)^{\alpha}}\left|\left\{(k, l) \in I_{r s}:\left|t_{k l p q}(x)-L\right| \geq \epsilon\right\}\right|
\end{aligned}
$$




$$
\begin{aligned}
& =h_{r s}^{\alpha} \frac{1}{\left(k_{r} l_{s}\right)^{\alpha}} \frac{1}{h_{r s}^{\alpha}}\left|\left\{(k, l) \in I_{r s}:\left|t_{k l p q}(x)-L\right| \geq \epsilon\right\}\right| \\
& \geq \frac{\beta_{1}}{1+\beta_{1}} \times \frac{\beta_{2}}{1+\beta_{2}} \frac{1}{h_{r s}^{\alpha}}\left|\left\{(k, l) \in I_{r s}:\left|t_{k l p q}(x)-L\right| \geq \epsilon\right\}\right| .
\end{aligned}
$$

Therefore, $\hat{S}_{\theta_{r s}}^{\alpha}-\lim _{(k, l) \rightarrow \infty} x(k, l)=L$.

Remark 2.1 The converse of this result is true for $\alpha=1$. However, for $\alpha<1$ it is not clear, and we leave it as an open problem.

Theorem 2.3 For any double lacunary sequence $\theta_{r s}, \hat{S}_{\theta_{r s}}^{\alpha} \subseteq \hat{S}_{2}^{\alpha}$ if $\lim \sup _{r} q_{r}^{\alpha}<\infty$ and $\lim \sup _{s} q_{s}^{\alpha}<\infty$.

Proof Suppose that $\lim \sup _{r} q_{r}^{\alpha}<\infty$ and $\lim \sup _{s} q_{s}^{\alpha}<\infty$. Then there exists $H>0$ such that $q_{r}^{\alpha}<H$ and $q_{s}^{\alpha}<H$ for all $r$ and $s$. Suppose that $x_{k l} \rightarrow L\left(S_{\theta_{r s}^{\alpha}}\right)$ and

$$
N_{r s}=\left|\left\{(k, l) \in I_{r s}:\left|t_{k l p q}(x)-L\right| \geq \epsilon\right\}\right|
$$

by the definition of $x_{k l} \rightarrow L\left(S_{\theta_{r s}}\right)$ given $\epsilon>0$, there exists $r_{0}, s_{0} \in N$ such that $\frac{N_{r s}}{h_{r s}^{\alpha}}<\epsilon$ for all $r>r_{0}$ and $s>s_{0}$. Let

$$
M:=\max \left\{N_{r s}: 1 \leq r \leq r_{0} \text { and } 1 \leq s \leq s_{0}\right\}
$$

Let $n$ and $m$ be such that $k_{r-1}<m \leq k_{r}$ and $l_{s-1}<n \leq l_{s}$. Therefore, we obtain the following:

$$
\begin{aligned}
& \frac{1}{(m n)^{\alpha}} \mid\left\{k \leq m \text { and } l \leq n:\left|t_{k l p q}(x)-L\right| \geq \epsilon\right\} \mid \\
& \leq \frac{1}{\left(k_{r-1} l_{s-1}\right)^{\alpha}} \mid\left\{k \leq k_{r} \text { and } l \leq l_{s}:\left|t_{k l p q}(x)-L\right| \geq \epsilon\right\} \mid \\
& \quad=\frac{1}{\left(k_{r-1} l_{s-1}\right)^{\alpha}}\left\{\sum_{i, j=1,1}^{r, s} N_{i, j}\right\} \\
& \leq \frac{M r_{0} s_{0}}{\left(k_{r-1} l_{s-1}\right)^{\alpha}}+\frac{1}{\left(k_{r-1} l_{s-1}\right)^{\alpha}}\left\{\sum_{i, j=r_{0}+1, r_{0}+1}^{r, s} N_{i, j}\right\} \\
& \leq \frac{M r_{0} s_{0}}{\left(k_{r-1} l_{s-1}\right)^{\alpha}}+\frac{1}{\left(k_{r-1} l_{s-1}\right)^{\alpha}}\left\{\sum_{i, j=r_{0}+1, r_{0}+1}^{r, s} \frac{N_{i, j} h_{i, j}^{\alpha}}{h_{i, j}^{\alpha}}\right\} \\
& \leq \frac{M r_{0} s_{0}}{k_{r-1} l_{s-1}}+\frac{1}{\left(k_{r-1} l_{s-1}\right)^{\alpha}}\left(\sup _{i, j \geq r_{0}, r_{0}} \frac{N_{i, j}}{h_{i, j}^{\alpha}}\right)\left\{\sum_{i, j=r_{0}+1, r_{0}+1}^{r, s} h_{i, j}^{\alpha}\right\} \\
& \leq \frac{M r_{0} s_{0}}{\left(k_{r-1} l_{s-1}\right)^{\alpha}}+\epsilon\left\{\sum_{i, j=r_{0}+1, r_{0}+1}^{r, s} h_{i, j}^{\alpha}\right\} \\
& \leq \frac{M r_{0} s_{0}}{\left(k_{r-1} l_{s-1}\right)^{\alpha}}+\epsilon H^{2} .
\end{aligned}
$$

This completes the proof of the theorem.

Theorem 2.4 Let $0<\alpha \leq \beta \leq 1$ and t be a positive real number, then $\hat{w}_{\theta_{r s}}^{\alpha}(t) \subseteq \hat{w}_{\theta_{r s}}^{\beta}(t)$. 
Proof Let $x=\left(x_{i j}\right) \in \hat{w}_{\theta_{r s}}^{\alpha}(t)$. Then given $\alpha$ and $\beta$ such that $0<\alpha \leq \beta \leq 1$ and a positive real number $t$ we write

$$
\frac{1}{h_{r s}^{\beta}} \sum_{(k, l) \in I_{r s}}\left|t_{k l p q}(x)-L\right|^{t} \leq \frac{1}{h_{r s}^{\alpha}} \sum_{(k, l) \in I_{r s}}\left|t_{k l p q}(x)-L\right|^{t},
$$

and we get that $\hat{w}_{\theta_{r s}}^{\alpha}(t) \subseteq \hat{w}_{\theta_{r s}}^{\beta}(t)$.

As a consequence of Theorem 2.4, we have the following.

Corollary 2.1 Let $0<\alpha \leq \beta \leq 1$ and $t$ be a positive real number. Then:

(i) If $\alpha=\beta$, then $\hat{w}_{\theta_{r s}}^{\alpha}(t)=\hat{w}_{\theta_{r s}}^{\beta}(t)$.

(ii) $\hat{w}_{\theta_{r s}}^{\alpha}(t) \subseteq \hat{w}_{\theta_{r s}}(t)$ for each $\alpha \in(0,1]$ and $0<t<\infty$.

Theorem 2.5 Let $\alpha$ and $\beta$ be fixed real numbers such that $0<\alpha \leq \beta \leq 1$ and $0<t<\infty$. If a sequence is a strongly $\hat{w}_{\theta_{r s}}^{\alpha}(t)$-summable sequence of order $\alpha$, to L, then it is $\hat{S}_{\theta_{r s}}^{\beta}$-statistically convergent of order $\beta$, to L, i.e., $\hat{w}_{\theta_{r s}}^{\alpha}(t) \subset \hat{S}_{\theta_{r s}}^{\beta}$.

Proof For any sequence $x=\left(x_{i j}\right)$ and $\epsilon>0$, we write

$$
\begin{aligned}
\sum_{(k, l) \in I_{r s}}\left|t_{k l p q}(x)-L\right|^{t} & =\sum_{\substack{(k, l) \in I_{r s} \\
\left|t_{k l p q}(x)-L\right| \geq \epsilon}}\left|t_{k l p q}(x)-L\right|^{t}+\sum_{\substack{(k, l) \in I_{r s} \\
\left|t_{k l p q}(x)-L\right|<\epsilon}}\left|t_{k l p q}(x)-L\right|^{t} \\
& \geq \sum_{\substack{(k, l) \in I_{r s} \\
\left|t_{k l p q}(x)-L\right| \geq \epsilon}}\left|t_{k l p q}(x)-L\right|^{t} \geq\left|\left\{(k, l) \in I_{r s}:\left|t_{k l p q}(x)-L\right| \geq \epsilon\right\}\right| \cdot \epsilon^{t}
\end{aligned}
$$

and so that

$$
\begin{aligned}
\frac{1}{h_{r s}^{\alpha}} \sum_{(k, l) \in I_{r s}}\left|t_{k l p q}(x)-L\right|^{t} & \geq \frac{1}{h_{r s}^{\alpha}}\left|\left\{(k, l) \in I_{r s}:\left|t_{k l p q}(x)-L\right| \geq \epsilon\right\}\right| \cdot \epsilon^{t} \\
& \geq \frac{1}{h_{r s}^{\beta}}\left|\left\{(k, l) \in I_{r s}:\left|t_{k l p q}(x)-L\right| \geq \epsilon\right\}\right| \cdot \epsilon^{t},
\end{aligned}
$$

this shows that if $x=\left(x_{i j}\right)$ is strongly $\hat{w}_{\theta_{r s}}^{\alpha}(t)$-summable sequence of order $\alpha$ to $L$, then it is $\hat{S}_{\theta_{r s}}^{\beta}$-statistically convergent of order $\beta$ to $L$. This completes the proof.

We have the following.

Corollary 2.2 Let $\alpha$ be fixed real numbers such that $0<\alpha \leq 1$ and $0<t<\infty$.

(i) If a sequence is strongly $\hat{w}_{\theta_{r s}}^{\alpha}(t)$-summable sequence of order $\alpha$ to $L$, then it is $\hat{S}_{\theta_{r s}}^{\alpha}$-statistically convergent of order $\alpha$ to L, i.e., $\hat{w}_{\theta_{r s}}^{\alpha}(t) \subset \hat{S}_{\theta_{r s}}^{\alpha}$.

(ii) $\hat{w}_{\theta_{r s}}^{\alpha}(t) \subset \hat{S}_{\theta_{r s}}$, for $0<\alpha \leq 1$.

\section{New sequence space}

In this section, we study the inclusion relations between the set of $\hat{S}_{\theta_{r s}}^{\alpha}$-statistical convergent sequences of order $\alpha$ and strongly $\hat{w}_{\theta_{r s}}^{\alpha}[M, t]$-summable sequences of order $\alpha$ with respect to an Orlicz function $M$. 
The study of Orlicz sequence spaces was initiated with a certain specific purpose in Banach space theory. Lindenstrauss and Tzafriri [24] investigated Orlicz sequence spaces in more detail, and they proved that every Orlicz sequence space $l_{M}$ contains a subspace isomorphic to $l_{p}(1 \leq p<\infty)$. The Orlicz sequence spaces are the special cases of Orlicz spaces studied in [25]. Orlicz spaces find a number of useful applications in the theory of nonlinear integral equations. Whereas the Orlicz sequence spaces are the generalization of $l_{p}$ spaces, the $l_{p}$-spaces find themselves enveloped in Orlicz spaces [26].

Recall in [25] that an Orlicz function $M:[0, \infty) \rightarrow[0, \infty)$ is continuous, convex, nondecreasing function such that $M(0)=0$ and $M(x)>0$ for $x>0$, and $M(x) \rightarrow \infty$ as $x \rightarrow \infty$.

An Orlicz function $M$ is said to satisfy $\Delta_{2}$-condition for all values of $u$, if there exists $K>0$ such that $M(2 u) \leq K M(u), u \geq 0$.

In the later stage different classes of Orlicz sequence spaces were introduced and studied by Parashar and Choudhary [27], Savaş [28-33] and many others.

Definition 3.1 Let $M$ be an Orlicz function, $t=\left(t_{k l}\right)$ be a sequence of strictly positive real numbers, and let $\alpha \in(0,1]$ be any real number. Now, we write

$$
\begin{aligned}
\hat{w}_{\theta_{r s}}^{\alpha}[M, t]= & \left\{x=\left(x_{k l}\right): P-\lim _{r s} \frac{1}{h_{r s}^{\alpha}} \sum_{(k, l) \in I_{r s}}\left[\frac{M\left(\left|t_{k l p q}(x)-L\right|\right)}{\rho}\right]^{t_{k l}}=0,\right. \\
& \text { uniformly in } p, q \text {, for some } L \text { and } \rho>0\} .
\end{aligned}
$$

If $x \in \hat{w}_{\theta_{r s}}^{\alpha}[M, t]$, then we say that $x$ is strongly double almost lacunary summable of order $\alpha$ with respect to the Orlicz function $M$.

If we consider various assignments of $M, \theta_{r s}$ and $t$ in the sequence spaces above, we are granted the following:

(1) If $M(x)=x, \theta=2^{r s}$, and $t_{k, l}=1$ for all $(k, l)$ then $\hat{w}_{\theta_{r s}}^{\alpha}[M, t]=\left[\hat{w}^{\alpha}\right]$.

(2) If $t_{k, l}=1$ for all $(k, l)$, then $\hat{w}_{\theta_{r s}}^{\alpha}[M, t]=\hat{w}_{\theta_{r s}}^{\alpha}[M]$.

(3) If $t_{k, l}=1$ for all $(k, l)$ and $\theta=2^{r s}$, then $\hat{w}_{\theta_{r s}}^{\alpha}[M, t]=\hat{w}^{\alpha}[M]$.

(4) If $\theta=2^{r s}$, then $\hat{w}_{\theta}^{\alpha}[M, t]=\hat{w}^{\alpha}[M, t]$.

In the followings theorems, we shall assume that $t=\left(t_{k l}\right)$ is bounded and $0<h=$ $\inf _{k l} t_{k l} \leq t_{k l} \leq \sup _{k l} t_{k l}=H<\infty$.

Theorem 3.1 Let $\alpha, \beta \in(0,1]$ be real numbers such that $\alpha \leq \beta$, and let $M$ be an Orlicz function, then $\hat{w}_{\theta_{r s}}^{\alpha}[M, t] \subset \hat{S}_{\theta_{r s}}^{\beta}$.

Proof Let $x \in \hat{w}_{\theta}^{\alpha}[M, t], \epsilon>0$ be given and $\sum_{1}$ and $\sum_{2}$ denote the sums over $(k, l) \in I_{r s}$, $\left|t_{k l p q}(x)-L\right| \geq \epsilon$ and $(k, l) \in I_{r s},\left|t_{k l p q}(x)-L\right|<\epsilon$, respectively. Since $h_{r s}^{\alpha} \leq h_{r s}^{\beta}$ for each $r, s$ we write

$$
\begin{aligned}
& \frac{1}{h_{r s}^{\alpha}} \sum_{(k, l) \in I_{r s}}\left[\frac{M\left(\left|t_{k l p q}(x)-L\right|\right)}{\rho}\right]^{t_{k l}} \\
& \quad=\frac{1}{h_{r s}^{\alpha}}\left[\sum_{1}\left[\frac{M\left(\left|t_{k l p q}(x)-L\right|\right)}{\rho}\right]^{t_{k l}}+\sum_{2}\left[\frac{M\left(\left|t_{k l p q}(x)-L\right|\right)}{\rho}\right]^{t_{k l}}\right] \\
& \geq \frac{1}{h_{r s}^{\beta}}\left[\sum_{1}\left[\frac{M\left(\left|t_{k l p q}(x)-L\right|\right)}{\rho}\right]^{t_{k l}}+\sum_{2}\left[\frac{M\left(\left|t_{k l p q}(x)-L\right|\right)}{\rho}\right]^{t_{k l}}\right]
\end{aligned}
$$




$$
\begin{aligned}
& \geq \frac{1}{h_{r s}^{\beta}}\left[\sum_{1}[M(\epsilon / \rho)]\right]^{t_{k l}} \\
& \geq \frac{1}{h_{r s}^{\beta}} \sum_{1} \min \left(\left[M\left(\epsilon_{1}\right)\right]^{h},\left[M\left(\epsilon_{1}\right)\right]^{H}\right), \quad \epsilon_{1}=\frac{\epsilon}{\rho} \\
& \geq \frac{1}{h_{r s}^{\beta}}\left|\left\{(k, l) \in I_{r s}:\left|t_{k l p q}(x)-L\right| \geq \epsilon\right\}\right| \min \left(\left[M\left(\epsilon_{1}\right)\right]^{h},\left[M\left(\epsilon_{1}\right)\right]^{H}\right) .
\end{aligned}
$$

Since $x \in \hat{w}_{\theta_{r s}}^{\alpha}[M, t]$, the left hand side of the inequality above tends to zero as $r, s \rightarrow \infty$ uniformly in $p, q$. Hence the right hand side tends to zero as $r, s \rightarrow \infty$ uniformly in $p, q$, and, therefore, $x \in \hat{S}_{\theta_{r s}}^{\beta}$. This proves the result.

Corollary 3.1 Let $\alpha \in(0,1]$ and $M$ be an Orlicz function, then $\hat{w}_{\theta_{r s}}^{\alpha}[M, t] \subset \hat{S}_{\theta_{r s}}^{\alpha}$.

We finally prove the following theorem.

Theorem 3.2 Let $M$ be an Orlicz function, and let $x=\left(x_{i j}\right)$ be a bounded sequence, then $\hat{S}_{\theta_{r s}}^{\alpha} \subset \hat{w}_{\theta_{r s}}^{\alpha}[M, t]$.

Proof Suppose that $x \in \ell_{2}^{\infty}$ and $\hat{S}_{\theta_{r s}}^{\alpha}-\lim x_{i j}=L$. Since $x \in \ell_{2}^{\infty}$, then there is a constant $K>0$ such that $\left|t_{k l p q}(x)\right| \leq K$. Given $\epsilon>0$, we write for all $p, q$

$$
\begin{aligned}
\frac{1}{h_{r s}^{\alpha}} \sum_{(k, l) \in I_{r s}}\left[M\left(\frac{\left|t_{k l p q}(x)-L\right|}{\rho}\right)\right]^{r_{k l}} & =\frac{1}{h_{r s}^{\alpha}} \sum_{1}\left[M\left(\frac{\left|t_{k l p q}(x)-L\right|}{\rho}\right)\right]^{r_{k l}} \\
& +\frac{1}{h_{r s}^{\alpha}} \sum_{2}\left[M\left(\frac{\left|t_{k l p q}(x)-L\right|}{\rho}\right)\right]^{r_{k l}} \\
& \leq \frac{1}{h_{r s}^{\alpha}} \sum_{1} \max \left\{\left[M\left(\frac{K}{\rho}\right)\right]^{h},\left[M\left(\frac{K}{\rho}\right)\right]^{H}\right\} \\
& +\frac{1}{h_{r s}^{\alpha}} \sum_{2}\left[M\left(\frac{\epsilon}{\rho}\right)\right]^{t_{k l}} \\
& \leq \max \left\{[M(T)]^{h},[M(T)]^{H}\right\} \\
& \times \frac{1}{h_{r s}^{\alpha}}\left|\left\{(k, l) \in I_{r s}:\left|t_{k l p q}(x)-L\right| \geq \epsilon\right\}\right| \\
& +\max \left\{\left[M\left(\epsilon_{1}\right)\right]^{h},\left[M\left(\epsilon_{1}\right)\right]^{H}\right\}, \quad \frac{K}{\rho}=T, \frac{\epsilon}{\rho}=\epsilon_{1} .
\end{aligned}
$$

Therefore, $x \in x \in \hat{w}_{\theta_{r s}}^{\alpha}[M, t]$. This proves the result.

\section{Competing interests}

The author declares that they have no competing interests.

\section{Acknowledgements}

The author would like to thank the referees for their help and useful discussions.

Received: 27 April 2013 Accepted: 22 July 2013 Published: 21 August 2013 


\section{References}

1. Fast, H: Sur la convergence statistique. Colloq. Math. 2, 241-244 (1951)

2. Schoenberg, IJ: The integrability of certain functions and related summability methods. Am. Math. Mon. 66, 361-375 (1959)

3. Fridy, JA: On statistical convergence. Analysis 5, 301-313 (1985)

4. Šalát, T: On statistical convergence of real numbers. Math. Slovaca 30, 139-150 (1980)

5. Cakalli, H: A study on statistical convergence. Funct. Anal. Approx. Comput. 1(2), 19-24 (2009)

6. Miller, $\mathrm{HI}$ : A measure theoretical subsequence characterization of statistical convergence. Trans. Am. Math. Soc. 347(5), 1811-1819 (1995)

7. Maddox, IJ: On strong almost convergence. Math. Proc. Camb. Philos. Soc. 85(2), 345-350 (1979)

8. Bhunia, S, Das, P, Pal, S: Restricting statistical convergence. Acta Math. Hungar. 134(1,2), 153-161 (2012)

9. Colak, R: Statistical convergence of order $\alpha$. In: Modern Methods in Analysis and Its Applications, pp. 121-129. Anamaya Pub., New Delhi (2010)

10. Colak, R, Bektas, CA: $\lambda$-Statistical convergence of order $\alpha$. Acta Math. Sci. Ser. B 31(3), 953-959 (2011)

11. Pringsheim, A: Zur theorie der zweifach unendlichen Zahlenfolgen. Math. Ann. 53, 289-321 (1900)

12. Móricz, F, Rhoades, BE: Almost convergence of double sequences and strong regularity of summability matrices. Math. Proc. Camb. Philos. Soc. 104, 283-294 (1988)

13. Lorentz, GG: A contribution to the theory of divergent sequences. Acta Math. 80, 167-190 (1948)

14. Mursaleen, M, Edely, OH: Statistical convergence of double sequences. J. Math. Anal. Appl. 288(1), $223-231$ (2003)

15. Basarir, M, Konca, S: On some lacunary almost convergent double sequence spaces and Banach limits. Abstr. Appl. Anal. 2012, Article ID 426357 (2012)

16. Cakan, C, Altaylý, B, Coskun, H: Double lacunary density and lacunary statistical convergence of double sequences. Studia Sci. Math. Hung. 47(1), 35-45 (2010)

17. Patterson, RF, Savas, E: Lacunary statistical convergence of double sequences. Math. Commun. 10, 55-61 (2000)

18. Savaş, E: Double almost statistical convergence of order $\alpha$. Adv. Differ. Equ. 2013, 62 (2013)

19. Fridy, JA, Orhan, C: Lacunary statistical convergence. Pac. J. Math. 160, 43-51 (1993)

20. Savaş, E, Patterson, RF: Some double lacunary sequence spaces defined by Orlicz functions. Southeast Asian Bull. Math. 35(1), 103-110 (2011)

21. Savaş, E: Remark on double lacunary statistical convergence of fuzzy numbers. J. Comput. Anal. Appl. 11(1), 64-69 (2009)

22. Savaş, E, Patterson, RF: Double sequence spaces defined by Orlicz functions. Iran. J. Sci. Technol., Trans. A, Sci. 31(2), 183-188 (2007)

23. Savaş, E, Patterson, RF: Double $\sigma$-convergence lacunary statistical sequences. J. Comput. Anal. Appl. 11(4), 610-615 (2009)

24. Lindenstrauss, J, Tzafriri, L: On Orlicz sequence spaces. Isr. J. Math. 101, 379-390 (1971)

25. Krasnoselskii, MA, Rutisky, YB: Convex Function and Orlicz Spaces. Noordhoff, Groningen (1961)

26. Kamthan, PK, Gupta, M: Sequence Spaces and Series. Dekker, New York (1980)

27. Parashar, SD, Choudhury, B: Sequence space defined by Orlicz function. Indian J. Pure Appl. Math. 25(14), 419-428 (1994)

28. Savas, $E:(A, \lambda)$-Double sequence spaces defined by Orlicz function and double statistical convergence. Comput. Math. Appl. 55(6), 1293-1301 (2008)

29. Savaş, E, Savaş, R: Some $\lambda$-sequence spaces defined by Orlicz functions. Indian J. Pure Appl. Math. 34(12), 1673-1680 (2003)

30. Savaș, E: On some new double lacunary sequences spaces via Orlicz function. J. Comput. Anal. Appl. 11(3), 423-430 (2009)

31. Savaş, E, Savaş, R: Some sequence spaces defined by Orlicz functions. Arch. Math. 40(1), 33-40 (2004)

32. Savaş, E, Patterson, RF: $(\sigma \lambda)$-Double sequence spaces via Orlicz function. J. Comput. Anal. Appl. 10(1), 101-111 (2008)

33. Savaş, E, Patterson, RF: Double sequence spaces defined by Orlicz functions. Iran. J. Sci. Technol., Trans. A, Sci. 31(2), 183-188 (2007) 